\title{
Correction to: Multiple model synchronization with multiary delta lenses with amendment and K-Putput
}

\author{
Zinovy Diskin $^{1} \oplus$, Harald König ${ }^{2}$ and Mark Lawford ${ }^{1}$ \\ ${ }^{1}$ McMaster University, Hamilton, Canada \\ ${ }^{2}$ University of Applied Sciences FHDW Hannover, Hannover, Germany
}

\section{Correction to: Formal Aspects of Computing (2019) 31: 611-640 https://doi.org/10.1007/s00165-019-00493-0}

Owing to a production error, the reference in footnote 6 to footnote 8 should instead refer to footnote 5 in the original article. There are also several missing symbols in Section 7.1 of the original article, and a corrected version of this section is provided on the following page.

\subsection{Categorification of corrs}

A distinctive feature of the framework developed in the paper is the triviality of the corr updates- they are just pairs $\left(R, R^{\prime}\right)$ of the old and the new corr (see Remark 1 on p. 626). However, in practice, new corrs would be computed incrementally with deltas rather than afresh. To make the framework closer to practice, we need to change the notion of a multimodel update $\mathrm{u}: \mathcal{A} \rightarrow \mathcal{A}^{\prime}$ and consider it to be a pair $\mathbf{u}=(u, r)$ with $u=\left(u_{1} . . u_{n}\right)$ a feet update and $r: R \rightarrow R^{\prime}$ a corr update rather than just a pair of states $\left(R, R^{\prime}\right)$. Then we would obtain a setting based on a category $\mathcal{R}$ of multimodels (that includes both local updates and corr updates) together with boundary projection functors $\boldsymbol{\partial}_{i}: \mathcal{R} \rightarrow \mathrm{A}_{i}, i=1 . . n$, which take a multimodel update $\mathbf{u}=\left(u_{1} \ldots u_{n}, r\right)$ and select its corresponding component, $\partial_{i}(\mathbf{u})=u_{i}$. For multiary update propagation, we require each projection $\partial_{i}$ to be the get-part of an asymmetric lens (with amendment) that for a given multimodel state $R$ puts any foot update $u_{i}: A_{i} \rightarrow A_{i}^{\prime}, A_{i}=\partial_{i}(R)$ back to a multimodel update $\mathbf{u}=\operatorname{put}_{i}^{R}\left(u_{i}\right): \mathcal{A} \rightarrow \mathcal{A}^{\prime}$. Note that as it includes all feet updates, operation put ${ }_{i}^{R}$ actually provides all local propagation operations ppg $_{i j}$ we considered in the paper: $\operatorname{ppg}_{i j}^{R}\left(u_{i}\right)=\boldsymbol{\partial}_{j}\left(\mathbf{p u t}_{i}^{R}\left(u_{i}\right)\right)$. The Putget law is the equality $\boldsymbol{\partial}_{i}(u)=u_{i} ; u_{i}^{@}$. In this way, a multiary symmetric lens is - by definition - a multiary span of asymmetric lenses $\left(\boldsymbol{\partial}_{i}\right.$, put $\left._{i}\right): \mathcal{R} \rightarrow \mathrm{A}_{i}, i=1 \ldots n$. Besides being better aligned with practice, the setting above would simplify notation and probably some technicalities, but its advantages were recognized when the paper was already written and submitted for reviewing. With a great regret, an accurate theory of the categorified version of multiary lenses is thus left for future work.

Publisher's Note Springer Nature remains neutral with regard to jurisdictional claims in published maps and institutional affiliations.

Published online 15 January 2020

Correspondence and offprint requests to: Z. Diskin, E-mail: diskinz@mcmaster.ca

The original article can be found online at https://doi.org/10.1007/s00165-019-00493-0. 